\title{
Análise comparativa de dados meteorológicos obtidos por estação convencional e automática em Londrina - PR
}

\section{Comparative analysis of meteorological data obtained by conventional and automatic stations in Londrina - PR}

\author{
Lívia Maria Pederzini Pereira $^{1 *}$; Paulo Henrique Caramori²; \\ Wilian da Silva Ricce ${ }^{3}$; João Henrique Caviglione ${ }^{4}$
}

\section{Resumo}

\begin{abstract}
O objetivo foi efetuar um estudo comparativo entre uma estação meteorológica convencional do IAPAR e outra automática pertencente ao SIMEPAR, localizadas lado a lado na estação experimental do IAPAR em Londrina - PR (latitude: $23^{\circ} 22^{\prime} \mathrm{S}$; longitude: $51^{\circ} 10^{\prime} \mathrm{W}$ e altitude: $585 \mathrm{~m}$ ). Foram analisados dados diários das seguintes variáveis meteorológicas: precipitação (Prec), temperaturas máxima (Tmax), mínima (Tmin) e média (Tmed) do ar, velocidade do vento (V) e umidade relativa do ar (URmed), durante o período de 1/ 10/1999 a 31/12/2006. Foram utilizados nas comparações dos dados os seguintes índices estatísticos: coeficiente de determinação $\left(\mathrm{R}^{2}\right)$; concordância (d); erro máximo (ME); erro absoluto médio (EAM); eficiência (EF); coeficiente de massa crítica (CRM); raiz quadrada do erro médio quadrático normalizado (RMSE); erros sistemático (Es) e aleatório (Ea). Com base nas equações de regressão linear verificou-se que houve significativa concordância para cada variável medida nas duas estações meteorológicas. As maiores diferenças foram encontradas na Tmax, URmed e Prec, com ME da ordem de $4,7^{\circ} \mathrm{C}, 24,5 \% \mathrm{e}$ $21,2 \mathrm{~mm}$, respectivamente. As análises realizadas evidenciaram que os dados obtidos na estação meteorológica automática possuem alta confiabilidade e podem ser utilizados em estudos climatológicos. Palavras-chaves: Estação meteorológica, análise comparativa, variáveis climáticas
\end{abstract}

\begin{abstract}
The objective was to compare variables collected with a conventional meteorological station of IAPAR with an automatic station of SIMEPAR, located side by side at the experimental station of IAPAR in Londrina - PR (latitude: $23^{\circ} 22^{\prime} \mathrm{S}$; longitude: $51^{\circ} 10^{\prime} \mathrm{W}$ and altitude: $585 \mathrm{~m}$ ). Daily data of the following meteorological variables were analyzed: rainfall (Prec), maximum (Tmax), minimum (Tmin) and mean (Tmed) temperature, wind speed (V), and mean relative humidity (UR med), during the period from 10/01/ 1999 to 12/31/2006. For data comparisons the following statistical indexes were used: determination coefficient $\left(\mathrm{R}^{2}\right)$; agreement (d); maximum error (EM); absolute mean error (EAM); efficiency (EF); critical mass coefficient (CRM); square root of the normalized quadratic mean error (RMSE); systematic (Es) and random (Ea) errors. Based on the linear regressions it was concluded that there was a significant agreement for each variable measured in both stations. The largest differences were found in the maximum temperature, mean relative humidity and rainfall, with $\mathrm{ME}$ of $4.7^{\circ} \mathrm{C}, 24.5 \%$ e $21.2 \mathrm{~mm}$, respectively. The analyses evidenced that data obtained with the automatic meteorological station have high quality and can be used in climatological studies.
\end{abstract}

Key words: Meteorological station, comparative analysis, climatological variables

1 Aluna de graduação do curso de Geografia, estagiária do IAPAR, Londrina - PR. E-mail: liviamppereira@gmail.com

2 Eng. Agrônomo, PhD., Pesquisador, Agrometeorologia, IAPAR, Londrina - PR.

3 Eng. Agrônomo, Mestre, Pesquisador, Agroconsult Ltda., Londrina - PR.

4 Eng. Agrônomo, Mestre, Pesquisador, Agrometeorologia, IAPAR, Londrina - PR.

* Autor para correspondência 


\section{Introdução}

Considerando-se que o clima é a variável mais importante na produção agrícola (AYOADE, 1986), a disponibilidade de dados meteorológicos confiáveis é fundamental para quantificar os impactos na produtividade das culturas. Grande parte dos dados sobre os elementos do clima é obtida através de estações meteorológicas convencionais (EMC) e automáticas (EMA). A diferença entre as duas estações é que a primeira exige a presença diária do observador para a coleta de dados, enquanto a segunda opera por meio de sensores eletrônicos que são captados por um sistema de aquisição de dados, denominado Datalogger, tendo como principal vantagem o registro contínuo de todos os elementos, com saídas dos dados em intervalos que o usuário programar (GALINA; VERONA, 2004).

$\mathrm{Na}$ agricultura, o monitoramento automático dos elementos meteorológicos tem contribuído não somente para o aumento da produtividade como, também, para a melhoria da qualidade dos produtos e para a preservação dos recursos naturais (SENTELHAS et al., 1997; TORRE NETO, 1995).

Alguns exemplos de aplicação no monitoramento das atividades agrícolas através de estações meteorológicas automáticas são: controle da irrigação de diferentes culturas (FERNANDES; SILVA; FOLEGATTI, 1995), monitoramento e previsão de geadas e ocorrência de pragas e doenças, entre outros.

A automatização na coleta de dados meteorológicos é uma tecnologia muito recente no país e por isso vem gerando discussões sobre a sua confiabilidade, custo e dificuldades de manutenção. Falhas no funcionamento dos equipamentos ocasionam frequentemente falhas na coleta de dados, havendo a necessidade da utilização de dados meteorológicos provenientes de estações convencionais. Por outro lado, esta tecnologia tende a substituir o processo convencional, em função das dificuldades de manutenção de pessoal pelas instituições que operam redes de estações. No entanto, a substituição das estações meteorológicas convencionais pelas automáticas exige cuidados, especialmente no que se refere à homogeneização da série de dados (SENTELHAS et al., 1997).

Trabalhos realizados por Sentelhas et al. (1997), Souza, Galvani e Assunção (2003) e Oliveira e Volpe (2004) mostraram através de suas análises boa concordância entre os elementos meteorológicos, ao compararem os dados obtidos pelas duas estações, principalmente em relação às variáveis de temperatura. Este trabalho teve por objetivo efetuar um estudo comparativo entre dados obtidos por EMA e EMC na cidade de Londrina-PR, em uma série de 86 meses de observações.

\section{Material e Métodos}

Os dados utilizados neste estudo foram obtidos na Estação Experimental do Instituto Agronômico do Paraná em Londrina - PR (latitude 23⒉'S, longitude $51^{\circ} 10^{\prime} \mathrm{W}$ e altitude $585 \mathrm{~m}$ ). O período analisado foi de 01/10/1999 a 31/12/2006, por meio de um monitoramento simultâneo das estações meteorológicas convencional (EMC) e automática (EMA), sendo avaliados valores diários dos seguintes elementos: precipitação, temperaturas máxima (Tmax), mínima (Tmin) e média (Tmed) do ar, velocidade do vento (v) e umidade relativa média do ar (URmed).

Os elementos meteorológicos temperatura e umidade relativa do ar foram coletados em condições distintas nas duas estações. Na estação convencional, seguindo o padrão da Organização Meteorológica Mundial, os sensores se localizaram dentro de abrigo termométrico de venezianas duplas e na automática em micro-abrigos de multiplacas, ambos na altura de 1,7m.

Os dados da Estação Automática foram coletados e armazenados por um datalogger (ref. Comercial Sutron modelo TS8210), com leitura dos sensores a cada minuto e armazenamento das médias em valores horários. A estação meteorológica convencional opera há 30 anos e a automática por 10 anos e ambas seguem as recomendações para as observações meteorológicas de acordo com a Organização Meteorológica Mundial. 
Os dias em que houve falha na coleta de dados em uma das estações foram eliminados. Por meio de visualização gráfica detectaram-se os dados suspeitos e retornou-se aos registros originais para correções. Em seguida, foi aplicada análise de regressão linear para cada variável e os seguintes índices estatísticos: concordância (d), erro máximo (ME), erro absoluto médio (EAM), raiz quadrada do erro médio quadrático normalizado (RMSE), eficiência (EF), coeficiente da massa residual (CRM), erros sistemático (Es) e aleatório (Ea), segundo Willmott, Ackelson e Davis (1985).

As características dos sensores utilizados em ambas as estações são apresentadas na tabela 1 e a tabela 2 apresenta a comparação na forma de obtenção dos dados meteorológicos absolutos e médios, a partir da EMC e da EMA.

Tabela 1. Variáveis analisadas das estações meteorológicas convencional (EMC) e automática (EMA), com respectivos sensores, fabricantes, sensibilidades/precisões e unidades de medidas.

\begin{tabular}{|c|c|c|c|c|c|}
\hline Estação & Variável & Elemento sensor & Fabricante & Precisão & $\begin{array}{l}\text { Unidades de } \\
\text { medida }\end{array}$ \\
\hline \multirow{6}{*}{ EMC } & Tmax & Mercúrio & R.FUESS & $0,1^{\circ} \mathrm{C}$ & ${ }^{\circ} \mathrm{C}$ \\
\hline & Tmin & Álcool & R.FUESS & $0,1^{\circ} \mathrm{C}$ & ${ }^{\circ} \mathrm{C}$ \\
\hline & Tmed & & & & ${ }^{\circ} \mathrm{C}$ \\
\hline & URmed & psicrômetro & R.FUESS & $0,1 \%$ & $\%$ \\
\hline & Prec. & Pluviômetro & & $0,1 \mathrm{~mm}$ & $\mathrm{~mm}$ \\
\hline & Vento & Anemógrafo & R.FUESS & $0.1 \mathrm{~m} \mathrm{~s}^{-1}$ & $\mathrm{~m} \mathrm{~s}^{-1}$ \\
\hline \multirow{6}{*}{ EMA } & Tmax & Termistor & Sutron & $0,1^{\circ} \mathrm{C}$ & ${ }^{\circ} \mathrm{C}$ \\
\hline & Tmin & Termistor & Sutron & $0,1^{\circ} \mathrm{C}$ & ${ }^{\circ} \mathrm{C}$ \\
\hline & Tmed & & & & ${ }^{\circ} \mathrm{C}$ \\
\hline & URmed & Capacitor & Sutron & $0,1 \%$ & $\%$ \\
\hline & Precip. & Báscula & Sutron & $0,2 \mathrm{~mm}$ & $\mathrm{~mm}$ \\
\hline & Vento & Anemômetro & Sutron & $0,1 \mathrm{~m} \mathrm{~s}^{-1}$ & $\mathrm{~m} \mathrm{~s}^{-1}$ \\
\hline
\end{tabular}

Tabela 2. Comparação da forma de obtenção dos dados meteorológicos absolutos e médios das estações meteorológicas convencional (EMC) e automática (EMA).

\begin{tabular}{|c|c|c|}
\hline Elemento & EMC & EMA \\
\hline \multirow{2}{*}{ Temperatura média } & $T \max +T \min +T_{9: 00}+2 T_{21: 00}$ & $\Sigma T_{i}^{(1)}$ \\
\hline & 5 & $\overline{24}$ \\
\hline Temperatura máxima & Leitura às 21:00h & $>$ valor das $24: 00 \mathrm{~h}$ \\
\hline Temperatura mínima & Leitura às 9:00h & $<$ valor das $24: 00 \mathrm{~h}$ \\
\hline \multirow{2}{*}{ Umidade Relativa } & $U R_{9: 00}+U R_{15: 00}+U R_{21: 00}$ & $\sum U R_{i}^{(1)}$ \\
\hline & 3 & 24 \\
\hline Velocidade do vento & Média diária & $\frac{\Sigma V_{i}^{(1)}}{24}$ \\
\hline Precipitação do dia $n+1$ & Leitura às 9:00 & So matório das chuvas ${ }^{(2)}$ \\
\hline
\end{tabular}

(1) Medidas obtidas a cada 1 hora ( $\mathrm{i}=00: 00 \mathrm{~h}, 01: 00 \mathrm{~h}, . .24: 00 \mathrm{~h}$.

(2) chuvas coletadas desde as 9:00h do dia $n$ até as 9:00h do dia $n+1$ 
Foram também utilizados os seguintes índices da EMA, $n$ o número de observações e $\hat{P}=\mathrm{a}+\mathrm{b} O_{i}$. estatísticos, conforme as equações 1,2,3,4,5,6,7 e 8. Se o valor medido na EMA for igual ao da EMC,

Em que $O_{i}$ são os dados coletados na EMA, $P_{i}$ então $\mathrm{ME}=\mathrm{EAM}=\mathrm{RMSE}=\mathrm{CRM}=0$ e $\mathrm{R}^{2}=\mathrm{d}=\mathrm{EF}=1$. os dados coletados na EMC, $\bar{O}$ as médias dos dados

$$
\begin{aligned}
& \text { Índice de concordância } \\
& d=1-\left[\sum_{i=1}^{n}\left(P_{i}-O_{i}\right)^{2} / \sum_{i=1}^{n}\left(\left|P_{i}-\bar{O}\right|+\left|O_{i}-\bar{O}\right|\right)^{2}\right] \\
& \text { Erro máximo: } \quad M E=\max \left(\left|O_{i}-P_{i}\right|\right)_{i=1}^{n} \\
& \text { Erro absoluto médio: } \\
& E A M=\left[\frac{1}{n} \sum_{i=1}^{n}\left(O_{i}-P_{i}\right)\right] \\
& \text { Raiz quadrada do erro médio quadrático } \\
& \text { normalizado: } \\
& R M S E=\left[\frac{1}{n} \sum_{i=1}^{n}\left(O_{i}-P_{i}\right)^{2}\right]^{0,5} \times\left(\frac{100}{\bar{O}}\right) \\
& \text { Eficiência: } \\
& E F=\left[\sum_{i=1}^{n}\left(O_{1}-\bar{O}\right)^{2}-\sum_{i=1}^{n}\left(O_{i}-P_{i}\right)^{2}\right] / \sum_{i=1}^{n}\left(O_{i}-\bar{O}\right)^{2}(5) \\
& \text { Coeficiente de massa residual } \\
& C R M=\left[\sum_{i=1}^{n} O_{i}-\sum_{i=1}^{n} P_{i}\right] / \sum_{i=1}^{n} O_{i} \\
& E_{s}=\left[\frac{1}{n} \sum_{i=1}^{n}\left(\hat{P}_{i}-O_{i}\right)^{2}\right]^{2} \\
& E_{a}=\left[\frac{1}{n} \sum_{i=1}^{n}\left(P_{i}-\hat{P}_{i}\right)^{2}\right]^{2}
\end{aligned}
$$

\section{Resultados e Discussão}

Os resultados obtidos das análises de regressão dos dados das temperaturas máxima, média e mínima do ar, umidade relativa, precipitação e velocidade do vento entre as estações automática e convencional são apresentados na Figura 1 e os índices estatísticos estão na Tabela 3. Todas as variáveis apresentaram ajuste significativo à regressão linear. Os valores do índice de concordância (d) foram superiores a 0,9 , exceto para a umidade relativa. Foram encontrados valores maiores para a estação convencional, ou seja, houve uma subestimativa da EMA em relação à EMC, com exceção da velocidade do vento.

Tabela 3. Indicadores estatísticos para a análise de concordância entre a variável Temperatura máxima (Tmax), Temperatura mínima (Tmín), Temperatura média (Tméd), Precipitação (Prec), Vel. vento (v) e Umidade relativa média do ar (URmed) obtidos em EMA e EMC em Londrina, PR no período de outubro/1999 a dezembro/2006.

\begin{tabular}{lccccccccc}
\hline \multicolumn{1}{c}{ Variável } & $\mathrm{R}^{2}$ & $\mathrm{~d}$ & $\mathrm{EAM}$ & $\mathrm{ME}$ & $\mathrm{RMSE}$ & $\mathrm{Es}$ & $\mathrm{Ea}$ & $\mathrm{EF}$ & $\mathrm{CRM}$ \\
\hline Tmax $\left({ }^{\circ} \mathrm{C}\right)$ & 0,993 & 0,991 & $-0,20$ & 4,72 & 1,47 & 0,167 & 0,010 & 0,9851 & $-0,0037$ \\
Tmín $\left({ }^{\circ} \mathrm{C}\right)$ & 0,995 & 0,995 & $-0,02$ & 1,97 & 1,77 & 0,083 & 0,001 & 0,9874 & $-0,0004$ \\
Tméd $\left({ }^{\circ} \mathrm{C}\right)$ & 0,975 & 0,977 & $-0,17$ & 4,67 & 2,83 & 0,358 & 0,007 & 0,9716 & $-0,0040$ \\
Prec. $(\mathrm{mm})$ & 0,984 & 0,976 & $-0,57$ & 21,24 & 46,86 & 2,875 & 0,280 & 0,9753 & $-0,0774$ \\
V. Vento $\left(\mathrm{m} / \mathrm{s}^{-1}\right)$ & 0,940 & 0,914 & 0,12 & 2,650 & 10,30 & 0,105 & 0,014 & 0,9174 & 0,0509 \\
URmed $(\%)$ & 0,89 & 0,775 & 9,22 & 24,5 & 13,19 & 352,0 & 80,44 & 0,6301 & 0,118 \\
\hline
\end{tabular}


A temperatura mínima (Figura 1c) foi o elemento que apresentou a melhor correlação das medidas entre o sensor automático e convencional, com boa precisão $\left(\mathrm{R}^{2}=0,9947\right)$ e exatidão $(\mathrm{a}=-0,113$ e $\mathrm{b}=1,0074) . \mathrm{A}$ EF de 0,9874 foi a mais elevada dentre os elementos analisados e o erro absoluto médio de $-0,02{ }^{\circ} \mathrm{C}$ foi inferior ao encontrado por Sentelhas et al. (1997) e superior ao de Cunha e Martins (2004), que encontraram valores de $0,41^{\circ} \mathrm{C}$ e $-0,33^{\circ} \mathrm{C}$, respectivamente. Houve uma pequena subestimativa da EMA de 0,04\% em relação à EMC.

A temperatura máxima diária (Figura 1a) apresentou uma boa exatidão ( $a=0,1962$ e $b=0,9966)$ e a segunda melhor precisão $\left(\mathrm{R}^{2}=0,9925\right) \mathrm{e}$ concordância $(\mathrm{d}=0,991)$. A EMC subestimou a EMA em $0,37 \%$. O erro máximo apresentou uma amplitude de $4,7^{\circ} \mathrm{C}$, e o erro absoluto médio foi de $-0,20^{\circ} \mathrm{C}$, diferente do valor encontrado por Oliveira e Volpe (2004) que obteve $1,00^{\circ} \mathrm{C}$, e de Sentelhas et al. (1997), que foi de $0,73^{\circ} \mathrm{C}$. Pode-se atribuir a diferença de valores encontrados nas estações aos tipos de sensores utilizados, sendo um termistor na EMA e um termômetro de mercúrio na EMC.

Para a temperatura média (Figura 1b), houve uma pequena dispersão dos dados com uma precisão de $\mathrm{R}^{2}=0,975$ e boa exatidão ( $\left.\mathrm{a}=0,0075 \mathrm{e} \mathrm{b}=1,0036\right)$, com um erro absoluto médio de $-0,17^{\circ} \mathrm{C}$, apontando maiores valores para a EMC. Entretanto o erro máximo da variável foi de $4,6^{\circ} \mathrm{C}$, próximo do valor de Sentelhas et al. (1997) de $4,3^{\circ} \mathrm{C}$ e diferente do valor de $6,04^{\circ} \mathrm{C}$ encontrado por Cunha e Martins (2004). Pode-se salientar que diferenças desta ordem são encontradas na temperatura média, devido aos procedimentos adotados no cálculo da média diária e nos tipos de sensores para cada estação.

A umidade relativa média (Figura 1f) foi o elemento analisado que mostrou as maiores diferenças entre a EMC e EMA, apresentando os maiores erros aleatórios e sistemáticos, devido à discrepância encontrada nos dados. Houve uma subestimativa de 13,38\% da EMA em relação à EMC, resultado contrário ao encontrado por Cunha e
Martins (2004) e Souza, Galvani e Assunção (2003) que encontraram valores maiores para a EMA. O erro médio absoluto foi de $9.2 \%$, valor superior aos $5,69 \%$ de Oliveira e Volpe (2004) e aos 2,9\% de Sentelhas et al. (1997).

As diferenças de valores na obtenção dos dados podem ocorrer devido ao tipo de elemento sensível dos instrumentos, que no caso da automática é um capacitor e na convencional um psicrômetro, e também a diferença na amostragem utilizada para se calcular a média da EMA e da EMC. Segundo Oliveira e Volpe (2004) é conveniente, ao se utilizar dados da EMA, muito critério na obtenção e manipulação, além de uma análise de consistência, por serem comuns valores de umidade relativa superiores a $100 \%$.

Com relação à precipitação pluviométrica (Figura 1d) verificou-se boa precisão $\left(R^{2}=0,984\right)$ e exatidão $(a=0,231$ e $b=1,0434)$, com o EAM da ordem de $0,57 \mathrm{~mm}$ e $\mathrm{d}=0,976$, entretanto houve uma pequena dispersão dos dados, devido aos erros sistemáticos e aleatórios. Houve uma tendência de valores menores da EMA em relação à EMC de 7\%. O valor da $\mathrm{ME}$ foi de 21,2mm, resultados próximos a esse foram encontrados por Cunha e Martins (2004) e Sentelhas e Caramori (2002), que atribuem a falha devido ao pluviômetro ser de báscula, pois há uma incapacidade do sensor de registrar todo o volume coletado na mesma velocidade com que ela ocorre em eventos de forte precipitação.

No caso da velocidade média do vento (Figura 1e) a análise de regressão mostrou que houve uma boa precisão $(\mathrm{R} 2=0,940)$ e exatidão $(\mathrm{a}=0,0907 \mathrm{e} \mathrm{b}=$ 0,9863 ) sendo o d igual a 0,91 . Diferente dos outros elementos apresentados neste trabalho, os dados da EMA foram maiores que na EMC, com a superestimativa de $5,4 \%$, mostrando que houve uma maior sensibilidade para a EMA. Os sensores utilizados são diferentes para a EMA e EMC, cada uma tem seu próprio momento de partida como de parada, fazendo com que exista um maior efeito da inércia no conjunto das canecas do anemógrafo da 


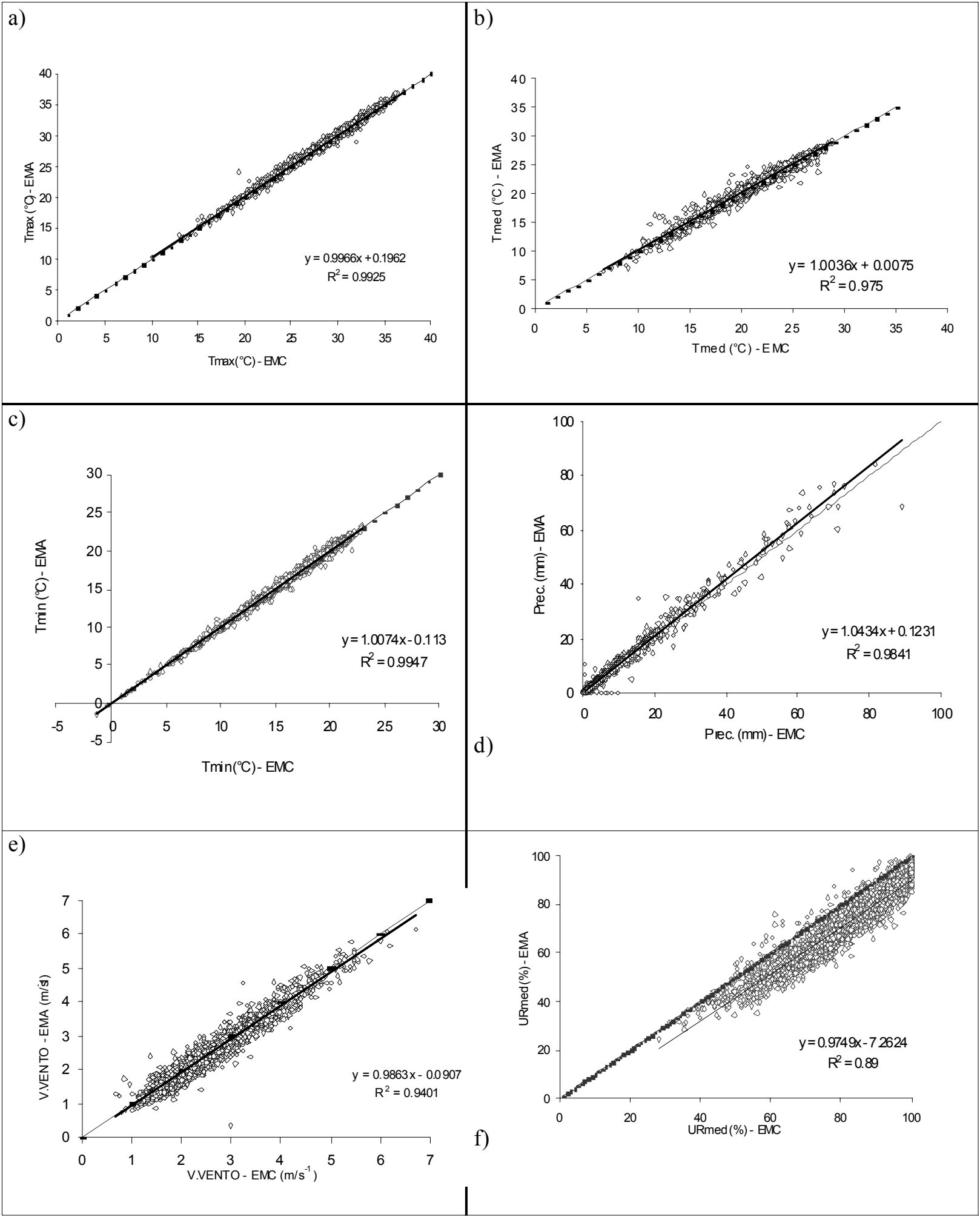

Figura 1. Relação entre dados meteorológicos diários observados na EMA e EMC: a)Temperatura máxima, b)Temperatura média, c)Temperatura mínima, d)Precipitação, e)Velocidade do vento, f) Umidade relativa média do ar no período de outubro-1999 a dezembro-2006, em Londrina - PR. 
EMC. O erro médio absoluto foi de $0,12 \mathrm{~ms}^{-1}$, valor inferior ao encontrado por Oliveira e Volpe (2004) que foi de $0,37 \mathrm{~m} / \mathrm{s}^{-1}$ e aos $0,71 \mathrm{~m} / \mathrm{s}^{-1}$ de Sentelhas et al. (1997). Para se obter uma alta confiabilidade dos dados da EMA, é necessário que os equipamentos tenham uma boa manutenção e calibração freqüente.

\section{Conclusão}

Os resultados em geral mostraram que houve boa concordância entre as estações meteorológicas, principalmente para as temperaturas máxima e mínima. A substituição da estação meteorológica convencional pela estação meteorológica automática não resultará em diferenças significativas em seus valores.

É necessária uma individual análise comparativa para cada localidade por um período expressivo antes de se realizar uma possível substituição. Entretanto no Brasil uma desativação da estação meteorológica convencional ainda não seria viável, devido ao elevado custo de manutenção que os equipamentos da estação meteorológica automática necessitam.

\section{Referências}

AYOADE, J. O. Introdução à climatologia para os trópicos. São Paulo: DIFEL, 1986.

CUNHA, A. R.; MARTINS, D. Estudo comparativo entre elementos meteorológicos obtidos em estações meteorológicas convencional e automática em Botucatu, SP, Brasil. Revista Brasileira de Agrometeorologia, Santa Maria, v. 12, n. 1, p. 103-111, 2004.
FERNANDES, A. L. T.; SILVA, F. C.; FOLEGATTI, M. V. Uso de estações automáticas para controle de irrigação no Estado de São Paulo. In: CONGRESSO BRASILEIRO DE AGROMETEOROLOGIA, 9., 1995, Campina Grande. Anais... Campina Grande: Sociedade Brasileira Agrometeorologia/UFPB, 1995. p. 1-3.

GALINA, M. H.; VERONA, J. A. Fontes de observações meteorológicas no estado de São Paulo. Estudos Geográficos, Rio Claro, v. 2, n. 1, p. 107-118, 2004.

OLIVEIRA, A. D.; VOLPE, C. A. Comparação de dados meteorológicos obtidos por estação convencional e automática. Revista Brasileira de Agrometeorologia, Santa Maria, v., n., p., 2004.

SENTELHAS, P. C.; CARAMORI, P. H. Inconsistências na medida da chuva com pluviômetros de báscula utilizados em estações meteorológicas automáticas. Revista Brasileira de Agrometeorologia, Santa Maria, v. 10, n. 2, p. 301-304.2002.

SENTELHAS, P. C.; MORAIS, S. O.; PIEDADE, S. M. S.; PEREIRA, A. R.; ANGELOCCI, L. R.; MARIN, F. R. Análise comparativa de dados meteorológicos obtidos por estação convencional e automática. Revista Brasileira de Agrometeorologia, Santa Maria, v. 5, n. 2, p. 215-221, 1997.

SOUZA, I. A.; GALVANI, E.; ASSUNÇÃO, H. F. Estudo comparativo entre elementos meteorológicos monitorados por estações convencional e automática na região de Maringá, Estado do Paraná. Acta Scientiarum, Maringá, v. 25, n. 2, p. 203-207, 2003.

TORRE NETO, A. Estudo e implementação de um sistema de monitoramento remoto de variáveis edafo-ambientais. 1995. Tese (Doutorado em Física) - Instituto de Física e Química de São Carlos, Universidade de São Paulo, São Carlos.

WILLMOTT, C. J.; ACKELSON, S. G.; DAVIS, R. E. Statistics for the evaluation and comparison of models. Journal of Geophysical Research, Ottawa, v. 90, n. C5 
